\title{
Porous calcium phosphate glass microspheres for orthobiologic applications
}

Kazi M. Zakir Hossain ${ }^{a}$,Uresha Patel ${ }^{a}$, Andrew R. Kennedy ${ }^{a l}$, Laura Macri-Pellizzeri ${ }^{b}$, Virginie Sottile $^{b}$, David M. Grant ${ }^{a}$, Brigitte E. Scammell ${ }^{c}$ and Ifty Ahmed ${ }^{l *}$

${ }^{a}$ Advanced Materials Research Group, Faculty of Engineering, University of Nottingham, UK

${ }^{\mathrm{b}}$ Wolfson STEM Centre, Division of Cancer and Stem Cells, School of Medicine, University of Nottingham, UK

${ }^{\mathrm{c}}$ Faculty of Medicine \& Health Sciences, Queen's Medical Centre, Nottingham, UK

${ }^{1}$ Current address: Engineering Department, Lancaster University, Lancaster, LA1 4YW, UK

*Corresponding authors: Ifty.Ahmed@nottingham.ac.uk

\begin{abstract}
Orthobiologics is a rapidly advancing field utilising cell-based therapies and biomaterials to enable the body to repair and regenerate musculoskeletal tissues. This paper reports on a costeffective flame spheroidisation process for production of novel porous glass microspheres from calcium phosphate based glasses to encapsulate and deliver stem cells. Careful selection of the glass and pore forming agent, along with a manufacturing method with the required processing window enabled the production of highly porous glass microspheres via a single-stage manufacturing process. The morphological and physical characterisation revealed porous microspheres with tailored surface and interconnected porosity (up to $76 \pm 5 \%$ ) with average pore size of $55 \pm 8 \mu \mathrm{m}$ and surface areas ranging from 0.34 to $0.9 \mathrm{~m}^{2} \mathrm{~g}^{-1}$. Furthermore, simple alteration of the processing parameters produced microspheres with alternate unique morphologies, such as with solid cores and surface porosity only. The tuneable porosity enabled control over their surface area, degradation profiles and hence ion release rates. Furthermore, biocompatibility of the microspheres was assessed using human mesenchymal stem cells (hMSCs) via direct cell culture experiments and analysis confirmed that they had migrated to within the centre of the microspheres. The novel microspheres developed have huge potential for tissue engineering and regenerative medicine applications.
\end{abstract}

Keywords: Calcium phosphate glass, porous microspheres, stem cells. 


\section{Introduction}

Orthobiologics is a rapidly advancing field which utilises cell-based therapies and biomaterials to promote healing. The development of orthobiologic solutions for repairing musculoskeletal disorders has provided clinicians alternatives to mechanical stabilisation, which has been the focus of orthopaedic procedures for many years. Orthobiologics have been utilised in a variety of different areas of medicine and their applications continue to expand [1] which is enabling orthobiologics to emerge as the frontline for disease treatment and has the potential to revolutionise medicine [2].

The advantages of using synthetic biomaterials over autograft and allograft bone materials are associated with their scale-up manufacturing and long term preservation in order to supply ondemand [3]. It was discovered that bioactive glasses could be used to stimulate osteogenesis, thereby leading to the concept of tissue regeneration. It has also since been discovered that the dissolution ion products from these glasses could also provide signals to the cells [4].

The three main types of bioactive glasses investigated for biomedical applications include silicates, borates and phosphate based glasses. Silicate based bioactive glasses are based on $\mathrm{SiO}_{2}$ (as the glass network former) and include other modifying oxides such as $\mathrm{Na}_{2} \mathrm{O}, \mathrm{CaO}$ and $\mathrm{P}_{2} \mathrm{O}_{5}$ (and were initially developed by the late Professor Larry Hench and commercialised as 45S5 Bioglass ${ }^{\mathrm{TM}}$ [5]). They have to a great extent been applied for use in hard tissue repair [57] and more recently have been added to toothpaste (Sensodyne ${ }^{\circledR}, N_{0}$ Namin $^{\mathrm{TM}}$, BioMin). Borate based glasses (where, $\mathrm{B}_{2} \mathrm{O}_{3}$ is the glass network former) degrade faster than silicatebased glasses and have also been investigated for biomedical applications [8]. Huang et al. [8] investigated the formulation $\mathrm{B}_{2} \mathrm{O}_{3} 46.1-\mathrm{CaO} 26.9-\mathrm{Na}_{2} \mathrm{O} 24.4-\mathrm{P}_{2} \mathrm{O}_{5} 2.6$ (in mol\%) and found that they could convert this glass into apatite following immersion within a dilute $(0.02 \mathrm{M})$ $\mathrm{K}_{2} \mathrm{HPO}_{4}$ solution. Phosphate based glasses are based on $\mathrm{P}_{2} \mathrm{O}_{5}$ (as the glass network former) and have been widely explored using $\mathrm{Na}_{2} \mathrm{O}$ and $\mathrm{CaO}$ including many other modifying oxides such as $\mathrm{CuO}$ [9], $\mathrm{Ag}_{2} \mathrm{O}$ [10], $\mathrm{TiO}_{2}$ [11], $\mathrm{SrO}$ [12], $\mathrm{MgO}$ [13] to provide enhanced properties or biological responses [14-18]. The main advantage of phosphate glasses over the silicate bioglasses and bioceramic materials, include their fully degradable and controllable resorption profiles (which can range from days/weeks/to many months), by simply tailoring the glass formulations [19, 20]. 
Microspheres (i.e. spherical particles) exhibit greater advantages over irregular-shaped particles such as improved flow properties, which would be particularly beneficial for biomedical applications (for example, by enabling improved delivery via minimally invasive surgical injection procedures [21]). Microparticles with hollow and/or porous morphologies have become extremely important in many scientific fields due to their advantageous functional properties. For example, hollow microspheres have been exploited as additives for adhesives and as fillers in construction composites due to their durability and light weight [22], whereas porous microparticles have been widely investigated for applications varying from stem cell research [23, 24], drug delivery [25], tissue engineering [26], separation sciences [27], supercapacitors [28] and energy storage [29]. More recently, hollow glass microspheres with porous walls (with porosity on the angstrom level) have been developed and are being investigated to store liquids and gases within their hollow interior with the aim to release them on demand [30]. However, the development of highly porous glass microspheres via a simple manufacturing process, with controlled surface porosity levels and with interconnected porosity (throughout the entire microsphere), could have the potential to totally revolutionise the microsphere industry by enabling many other applications for these materials.

Manufacturing processes for porous materials depend heavily on the type of material used. For example, techniques utilised for making porous glass and/or ceramic scaffolds commonly use methods such as incorporation of a removable space holder [31], polymer foam replication [32], sol-gel [33], gel cast foaming [34] and solid freeform methods (i.e. 3D printing) [35]. These manufacturing processes involve multiple processing steps which can be laborious, time consuming and energy intensive. The dissolution and thermal decomposition of space holders and sacrificial polymer templates can also result in residual contaminants which not only affects further processing but also the mechanical and physical properties of the resultant porous material. In the case of solid freeform fabrication techniques (3D printing), maintaining the viscosity of the ink (glass particles along with the polymer binder) is critical and can be time consuming requiring several steps of printing, drying and sintering [35].

Processes utilised for manufacturing polymer based porous microspheres have mostly been via emulsion-solvent evaporation, spray drying and phase separation techniques [25, 36, 37]. Whilst, ceramic microspheres have commonly been fabricated via gelation, emulsification and precipitation processes [38, 39] and solid (non-porous) and hollow glass microspheres have been fabricated via sol-gel [40] and flame spheroidisation [41] processes. However, when considering the manufacture of porous glass microspheres with fully controlled surface and 
fully interconnected porosity, the main challenges have included high production costs alongside lengthy and/or complex manufacturing processes, scale-up limitations and control over porosity size, interconnected porosity and morphology.

In this paper, the authors report a new and cost-effective process for manufacturing highly porous calcium phosphate $(\mathrm{CaP})$ glass microspheres with tailorable surface and fully interconnected porosity (up to $76 \%$ ) within each microsphere produced, via a single-stage flame spheroidisation process. Preliminary biological analyses were performed via direct cell culture studies using human mesenchymal stem cells including the suitability of these microspheres to support cell adhesion and growth.

\section{Materials and methods}

\subsection{Manufacture of solid and porous calcium phosphate glass microspheres}

Calcium phosphate glass in the system $40 \mathrm{P}_{2} \mathrm{O}_{5} \cdot 16 \mathrm{CaO} \cdot 24 \mathrm{MgO} \cdot 20 \mathrm{Na}_{2} \mathrm{O}(\mathrm{mol} \%$ denoted as P40) was prepared using $\mathrm{NaH}_{2} \mathrm{PO}_{4}, \mathrm{CaHPO}_{4}, \mathrm{MgHPO}_{4}$ and $\mathrm{P}_{2} \mathrm{O}_{5}$ (Sigma-Aldrich, UK) as starting materials. These precursors were placed into a $100 \mathrm{ml}$ volume $\mathrm{Pt} / 5 \% \mathrm{Au}$ crucible and dried at $350^{\circ} \mathrm{C}$ for 30 minutes in a furnace. The sample was then melted at $1150^{\circ} \mathrm{C}$ for 90 minutes. The molten glass was then quenched onto a steel plate and allowed to cool to room temperature.

Once cooled, the glass was ground into microparticles using a Retsch PM100 milling machine and sieved into varying size ranges spanning 63-300 $\mu \mathrm{m}$. The solid non-porous microspheres were produced using the flame spheroidisation method which utilised an oxy/acetylene flame spray gun (MK 74, Metallisation Ltd, UK). Porous glass microspheres were produced by introducing porogens to the glass feed, which were then fed simultaneously into the thermal spray gun. Post manufacture, the microspheres were collected from cooling trays and were then subjected to an acid wash step by gently stirring them in acetic acid (5M) for 2 min followed by washing using deionised water (for $5 \mathrm{~min}$ ) and then dried in an oven at $50^{\circ} \mathrm{C}$ for $24 \mathrm{~h}$.

\subsection{Characterisation methods}

\subsubsection{Scanning electron microscopy (SEM) and energy dispersive $X$-ray (EDX) analysis}

The surface and cross-sectional morphology was qualitatively examined using scanning electron microscopy (SEM - Philips XL30, FEI, USA) operated at 20kV. Cross sections were achieved by embedding the microspheres in a cold set epoxy resin and polished with SiC paper 
followed by a diamond cloth. Quantitative compositional analysis of the $\mathrm{CaP}$ microspheres was carried out using an energy dispersive X-ray (EDX, INCA Oxford Instruments) detector at an accelerating voltage of $20 \mathrm{kV}$ and a working distance of $10 \mathrm{~mm}$. All samples were carbon coated using an evaporation coater (Edwards coating System E306A) in order to avoid image distortion due to charging.

\subsubsection{Mercury porosimetry and BET surface area analysis}

The porosity of the microspheres was investigated using mercury intrusion porosimetry (Micromeritics Autopore IV 9500). A 5cc powder penetrometer (Micrometrics) with 1cc intrusion volume was used for all samples. An empty penetrometer test was also conducted as a blank before running the samples.

The surface area was determined via gas adsorption using the BET technique (Micrometrics Gemini 2360) where approximately $0.5 \mathrm{~g}$ of the microspheres were initially degassed under nitrogen at $110^{\circ} \mathrm{C}$ for at least $2 \mathrm{~h}$ before performing multipoint BET analysis.

\subsubsection{Degradation and ion release studies}

Degradation analyses of the microspheres was evaluated by means of mass loss measurements in ultra-purified water (Milli-Q) $(1 \% \mathrm{w} / \mathrm{v})$ at $37^{\circ} \mathrm{C}$ over 28 days (the medium was refreshed every 2 days). The degraded microspheres were removed from the medium at various time points $(1,3,7,14,21$, and 28 days) and placed onto tissue paper to blot dry then weighed immediately.

$$
\operatorname{Mass} \operatorname{Loss}(\%)=\frac{M_{0}-M_{t}}{M_{0}}
$$

Where $M_{o}$ is the initial mass and $M_{t}$ is the mass at test time point $\mathrm{t}$.

Ion release profiles of the microspheres was conducted in Dulbecco's Modified Eagle Medium (DMEM, Sigma-Aldrich, UK) cell culture media (1\% w/v) over 14 days (the media was again refreshed every 2 days) using inductively coupled plasma mass spectrometry (ICP-MS, Thermo-Fisher iCAP-Q model).

\subsubsection{Cell culture study}

\subsubsection{Preparation of CaP microspheres extract}


Porous CaP microspheres were sterilised through two 15-min wash steps using $70 \%$ EtOH. After complete evaporation of $70 \% \mathrm{EtOH}$ at room temperature in sterile conditions, porous microspheres were soaked in standard cell culture (SC) medium (low glucose DMEM (Gibco) supplemented with $10 \%$ foetal calf serum (Gibco), $1 \%$ penicillin and streptomycin, $1 \% \mathrm{~L}-$ Glutamine, $1 \%$ of non-essential amino acids) at a final concentration of $100 \mathrm{mg} \mathrm{ml}^{-1}$ at $37^{\circ} \mathrm{C}$ and $5 \% \mathrm{CO}_{2}$. After $24 \mathrm{~h}$, the conditioned medium containing the microsphere ion extracts was collected, filtered using $0.22 \mu \mathrm{m}$ syringe filters and used to prepare three serial dilutions with 1:10 ratio using SC medium.

\subsubsection{MTT assay}

The evaluation of cytotoxicity was performed using a "Cell Growth Determination Kit, MTT based" (Sigma Adlrich, UK); for this purpose, 1x10 4 immortalised human bone marrowderived mesenchymal stem cells (hMSCs) were seeded in 96-well plate in $100 \mu \mathrm{l}$ of SC medium. After 24 h, SC medium was replaced with ion extract conditioned medium or the 3 serial dilutions prepared as shown in section 2.2.4.1. Moreover, SC medium alone or supplemented with $5 \%$ of DMSO were included as controls. After $24 \mathrm{~h}$, cells were washed once with PBS and incubated during $3 \mathrm{~h}$ at $37^{\circ} \mathrm{C}$ and $5 \%$ of $\mathrm{CO}_{2}$ with a $\mathrm{SC}$ medium supplemented with $10 \%$ of MTT solution. After incubation, media was aspirated and $100 \mu$ of DMSO was added to each well and incubated for $15 \mathrm{~min}$ in gentle shaking to facilitate the formazan salts dissolution. Absorbance was read using a plate reader at $570 \mathrm{~nm}$, setting $650 \mathrm{~nm}$ as reference. The cell viability percent was calculated in comparison to SC medium control using the following formula:

$$
\text { Viability }(\%)=\frac{O D 570 \mathrm{~s}}{O D 570 c}
$$

Where, $O D 570$ s and $O D 570 \mathrm{c}$ are the optical densities measured in the samples of interest and in the control, respectively.

\subsubsection{Direct seeding of cells on porous CaP microspheres}

For direct seeding of cells onto the porous $\mathrm{CaP}$ microspheres, $2 \times 10^{4}$ immortalized GFP-labelled human bone marrow-derived mesenchymal stem cells (hMSCs) were seeded on $10 \mathrm{mg}$ of sterile microspheres into low-adherent 48 -well plates previously coated with $1 \%$ (w/v) solution of poly(2-hydroxyethyl methacrylate) (poly-HEMA) and Ethanol 95\% in SC medium. Cells were cultured by 14 days at $37^{\circ} \mathrm{C}$ and $5 \% \mathrm{CO}_{2}$. The media was refreshed every $48 \mathrm{~h}$. 


\subsubsection{Presto Blue assay}

Metabolic activity of cells seeded on porous $\mathrm{CaP}$ glass microspheres was analysed using Presto Blue reagent at day 2, day 5 and day 12 according to the manufacturer's indications. Briefly, cells were incubated with a solution of SC medium supplemented with $10 \%$ of Presto Blue at $37^{\circ} \mathrm{C}$ for $40 \mathrm{~min}$. The fluorescence was then measured at $560 \mathrm{~nm}$ and $590 \mathrm{~nm}$ as excitation and emission wavelengths using a plate reader (Tecan infinite 200).

\subsubsection{Cell staining and imaging}

Cell imaging was performed at day 14 in paraformaldehyde-fixed cells. For Environmental Scanning Electron Microscopy (ESEM), post fixed cells were washed twice with distilled water and analysed using a FEI Quanta 650 ESEM microscope.

For confocal laser scanning microscopy analysis, cell nuclei were counterstained with $10 \mu \mathrm{g}$ $\mathrm{ml}^{-1}$ of Hoechst 33258 for $10 \mathrm{~min}$ and cytoskeletal actin fibres were visualised using Vectashield mounting medium containing TRITC-Phalloidin (Vector Laboratories, UK). Confocal laser scanning microscopy was carried out on a Zeiss LSM 880 microscope using a a 10x 0.3 NA or 20x 0.5 NA objectives setting $5.77 \mu \mathrm{m}$ and $1.77 \mu \mathrm{m}$ spacing respectively and sequential imaging channels for each fluorophore. The following lasers were assigned to each fluorophore: Hoechst: $\lambda_{\text {ex. }} 405 \mathrm{~nm}$ laser, $\lambda_{\text {em. }}$ 405-437 nm, FITC-GFP: $\lambda_{\text {ex. }} 488 \mathrm{~nm}$ laser, $\lambda_{\text {em. }}$ 496-556 nm, TRITC-Phalloidin: $\lambda_{\text {ex. }} 561 \mathrm{~nm}$ laser, $\lambda_{\text {em. }} 564-600 \mathrm{~nm}$.

\subsubsection{Statistical analysis}

For the cell study, one biological replicate was included in each assay. Data is presented as mean \pm SEM. Statistical analysis was performed using GraphPad PRISM 7.01 software package via the One-way ANOVA with Tukey's multiple comparison post hoc test. A 95\% confidence level was considered significant.

\section{Results}

Both solid (non-porous) and highly porous $\mathrm{CaP}$ glass microspheres were produced via the flame spheroidisation process as shown in Figure 1. Figure 1a shows uniform non-porous glass microspheres with an average diameter of $108( \pm 10) \mu \mathrm{m}$. Figure $1 \mathrm{~b}$ represents the yield and distribution of pore morphologies achieved utilising the novel manufacturing process 
developed herein. Figure 1c shows a SEM image of a porous CaP glass microsphere with a wide distribution of smaller surface pores (ranging from macroporous sized features to $30 \mu \mathrm{m}$ ). Whilst Figures $1 \mathrm{~d}+\mathrm{e}$ highlights porous $\mathrm{CaP}$ glass microspheres with a combination of larger (between 50 - $80 \mu \mathrm{m}$ ) and smaller (from 5 - $20 \mu \mathrm{m}$ ) surface pores. Comparatively larger surface pores with similar internal larger interconnected pores were also produced (as shown in Figure 1e).
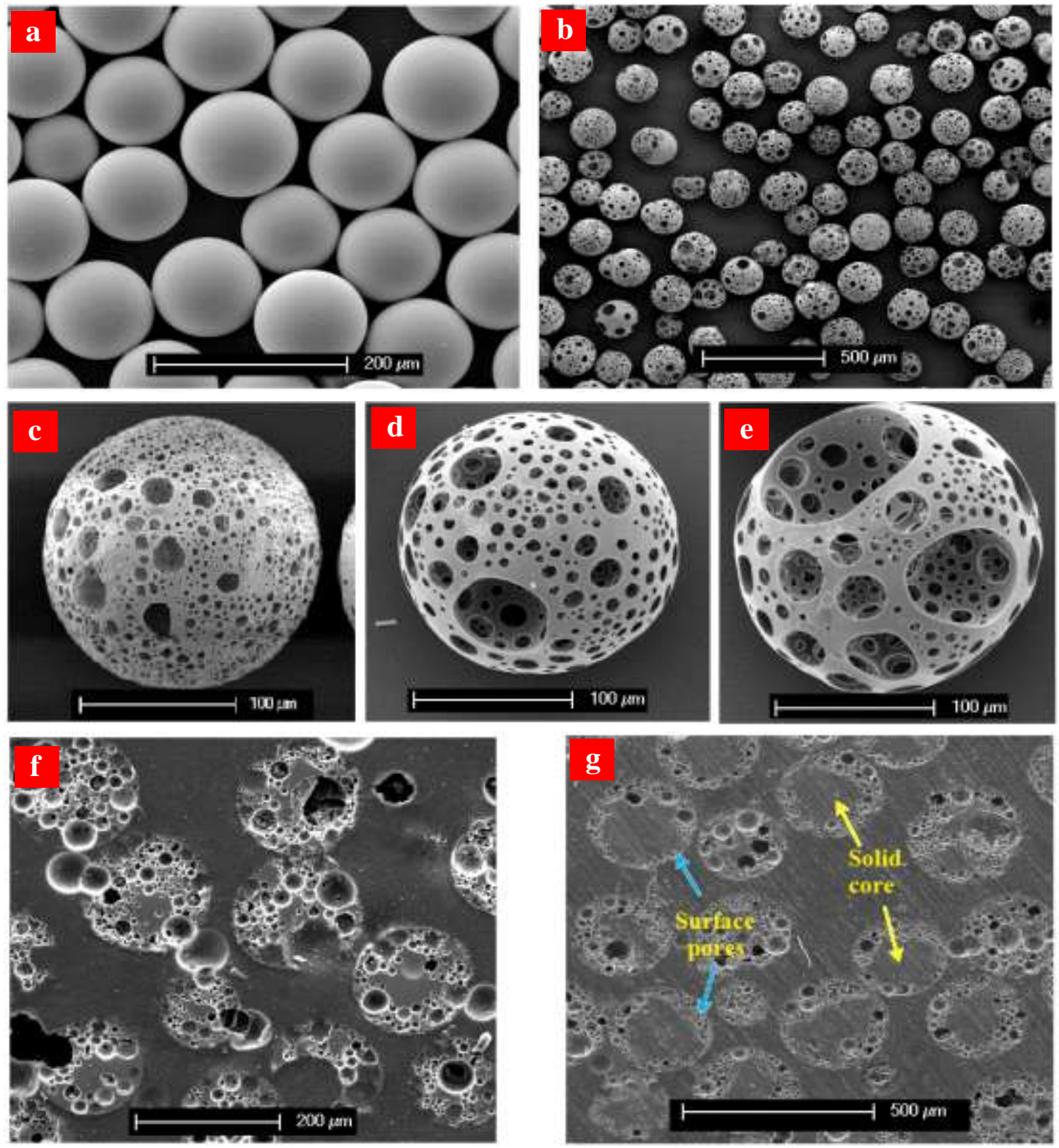

Figure 1: Representative SEM images of solid (non-porous) and porous CaP glass microspheres: a) Shows the distribution and yield of solid microspheres, b) Scale-up production of porous glass microspheres, $\boldsymbol{c}$ ) Surface morphology of porous glass microsphere with smaller surface pores, $\boldsymbol{d})$ Porous glass microsphere with a mixture of small and large 
surface pores, e) Porous glass microsphere with comparatively larger surface pores, $\boldsymbol{f}$ ) Crosssectional SEM images of resin embedded porous CaP glass microspheres with interconnected open porosity, and $\mathbf{g}$ ) Microspheres with surface pores (indicated with blue arrows) and solid core (indicated by yellow arrows).

The cross-sectional SEM images of the porous microspheres (as shown in Figure 1f) revealed the inner porosity and interconnectivity of the pores, which showed that the microspheres consisted mainly of smaller pores embedded within larger pores. Microspheres with surface porosity and a solid core were also manufactured as can be seen in Figure 1g when altering manufacturing parameters i.e. size of initial particles.

Characterisation of the $\mathrm{CaP}$ microspheres revealed posority levels of up to and over $76( \pm 5) \%$ could be achived depending on the process parameters. Mercury porosimetry analysis of porous and solid $\mathrm{CaP}$ glass microspheres is shown in Figure 2a. The mean pore size distibution for the porous microspheres was determined to be approxiamtely $55( \pm 8) \mu \mathrm{m}$ and also revealed inner pore features at the submicron and nano porosity levels (as seen in Figure 2b). Figure 2c highlights the percentage porosity change with increasing glass powder/porogen ratio content. The porosity of the compact solid microspheres (with a size range of between 100-125 $\mu \mathrm{m}$ ) was found to be approximaltely $37( \pm 4) \%$ where the interparticulate gaps between the solid microspheres were characterised as pores. However, for the porous glass microspheres approximately $76( \pm 5) \%$ porosity levels were obtained. The surface area (ascertained via BET analysis) for the non-porous $\mathrm{CaP}$ microspheres was $0.09( \pm 0.01) \mathrm{m}^{2} \mathrm{~g}^{-1}$ and the porous $\mathrm{CaP}$ microspheres were found to have an order of magnitude higher surface area which ranged from between $0.34( \pm 0.02)$ to $0.90( \pm 0.03) \mathrm{m}^{2} \mathrm{~g}^{-1}$ dependant on the micropshere size range selected (see Figure 2d). For example, the lower diameter size range porous microspheres (i.e between 63-125 $\mu \mathrm{m}$ ) revealed a significant number of larger surface pores (hence a lower surface area of $\left.0.34 \mathrm{~m}^{2} \mathrm{~g}^{-1}\right)$. Whilst, a comparatively higher surface area $\left(0.9 \mathrm{~m}^{2} \mathrm{~g}^{-1}\right)$ was obtained for the larger diameter size range porous microspheres (i.e. between 200-300 $\mu \mathrm{m}$ ) which revealed a larger number of smaller surface pores. 

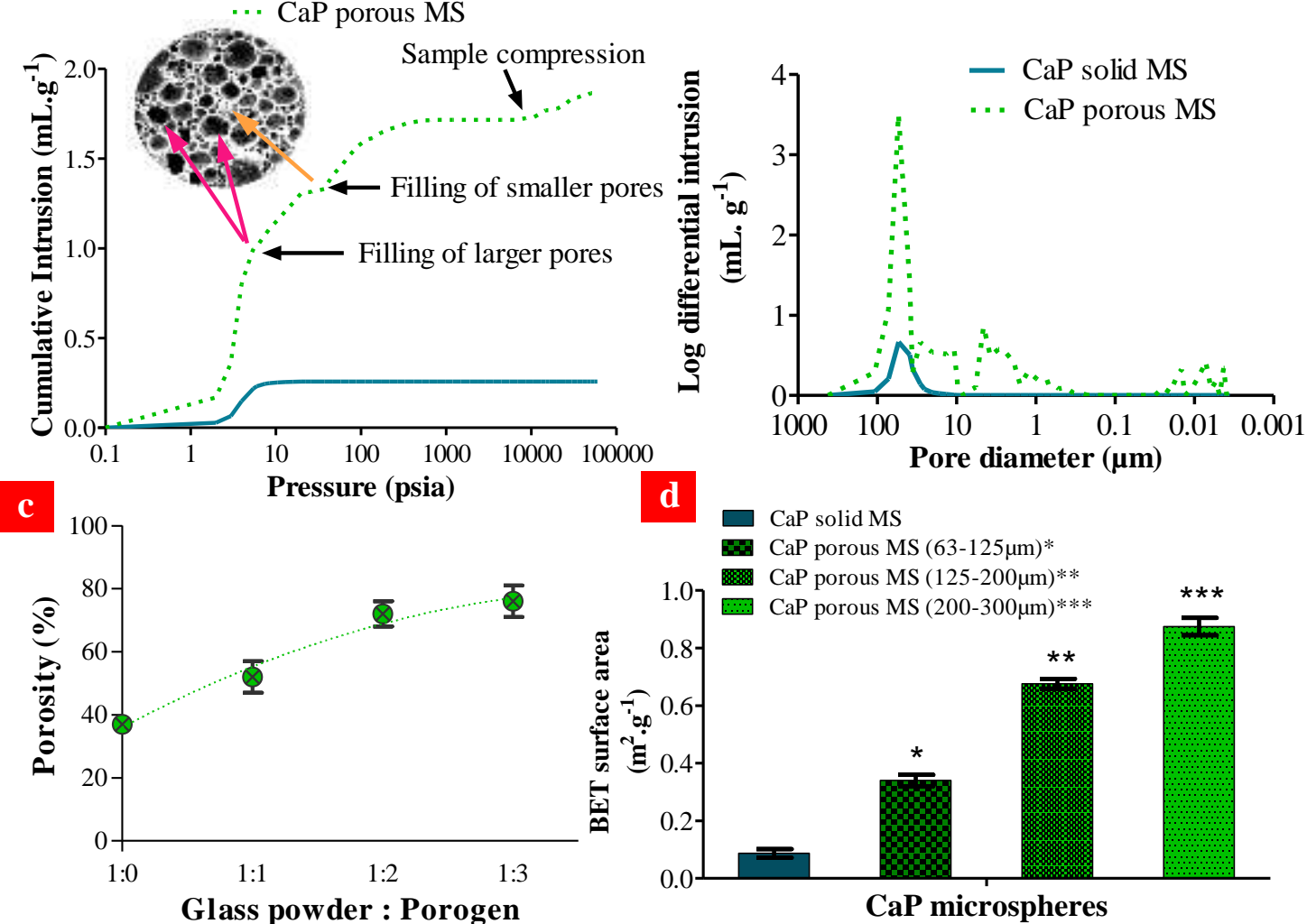

d CaP solid MS

CaP porous MS $(63-125 \mu \mathrm{m}) *$

CaP porous MS $(125-200 \mu \mathrm{m}) * *$

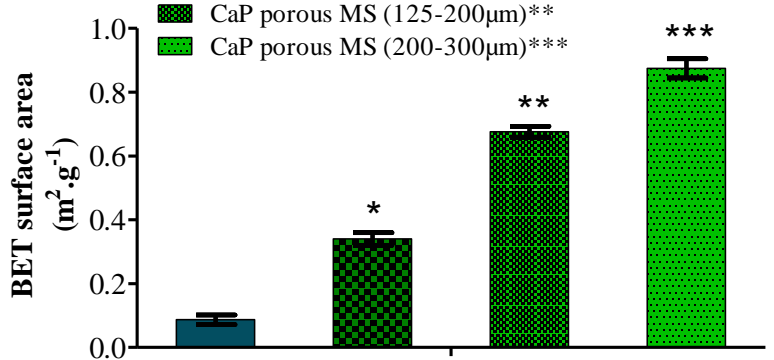

CaP microspheres

Figure 2: Porosity and surface area properties of CaP glass microspheres: a) Mercury intrusion curves of the solid and porous CaP glass microspheres, $\boldsymbol{b})$ log differential intrusion curves of solid and porous CaP glass microspheres highlighting the range of pore sizes achieved, c) Porosity (\%) of microspheres obtained with increasing glass powder/porogen ratio content and d) BET surface area analysis of the solid and porous CaP glass microspheres (between 63 to $300 \mu$ m diameter ranges).

EDX analysis confirmed negligible changes in glass compositions before and after spheroidisation (see Figure 3a), with only slight localised increases in Ca content which were mainly observed around the pores created.

Porous $\mathrm{CaP}$ microspheres revealed an approximate $30 \%$ mass loss compared to $9 \%$ mass loss for the solid non-porous microspheres over a 28 day degradation period in ultra-purified water at $37^{\circ} \mathrm{C}$ (see Figure $3 b$ ). Figures $3 c-f$ show increased ion release profiles (for example, $\mathrm{Ca}^{2+}$ $\sim 47( \pm 3)$ ppm, $\mathrm{PO}_{4}{ }^{3-} \sim 49( \pm 3) \mathrm{ppm}$, and $\mathrm{Mg}^{2+} \sim 20( \pm 3)$ ppm per day) for the porous CaP glass microspheres (size range 125-200 $\mu \mathrm{m})$ compared to the solid non-porous microspheres $\left(\mathrm{Ca}^{2+}\right.$ $\sim 45( \pm 1)$ ppm, $\mathrm{PO}_{4}{ }^{3-} \sim 34( \pm 2) \mathrm{ppm}$, and $\mathrm{Mg}^{2+} \sim 13( \pm 1)$ ppm per day) when degraded in 
standard cell culture medium (DMEM) at $37^{\circ} \mathrm{C}$ for 14 days, which was attributed to their higher surface area.
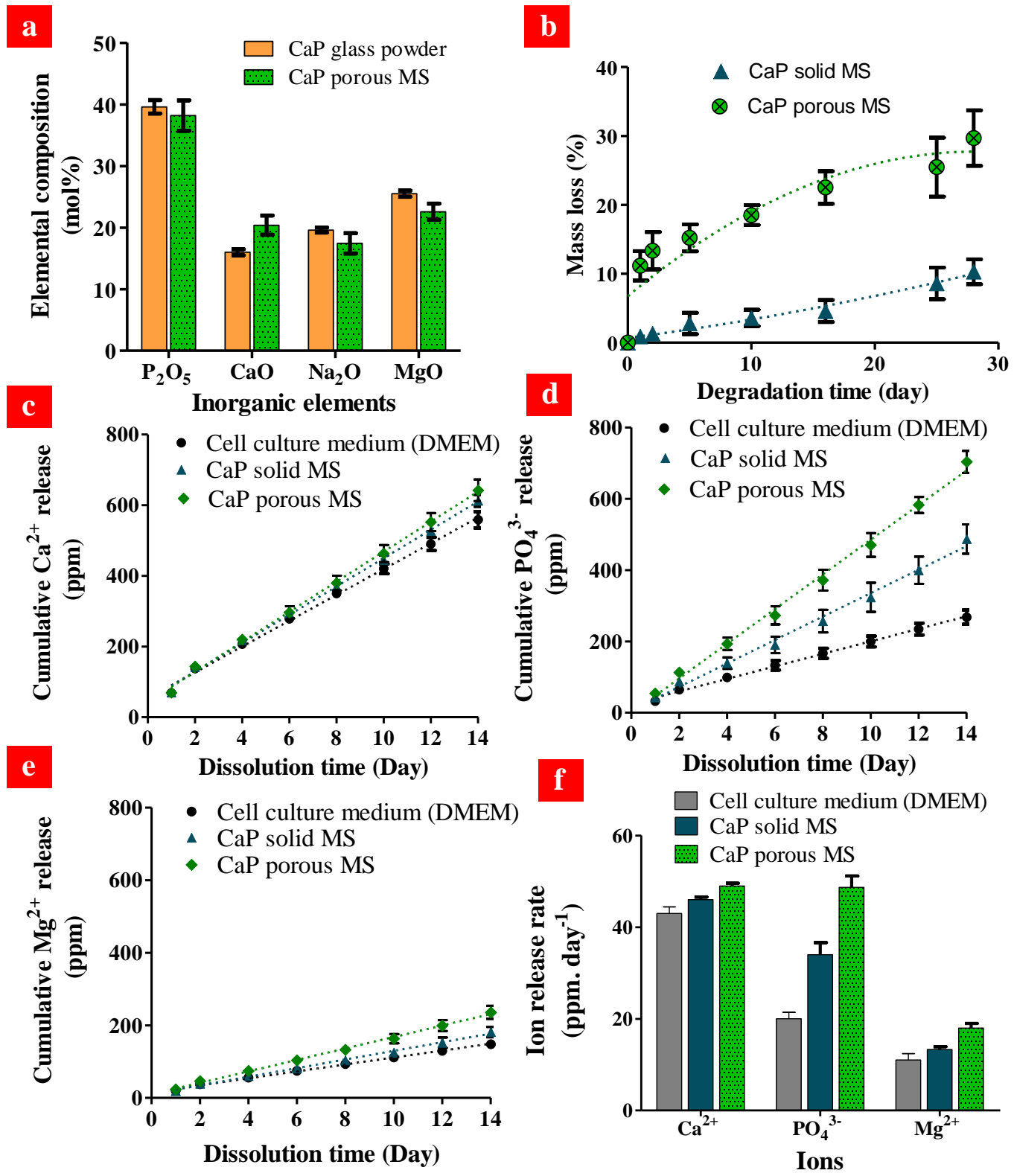

Figure 3: Chemical composition, degradation and ion release profiles of CaP glass microspheres: a) Chemical composition of the glass (ascertained via EDX analysis) before and after spheroidisation, b) Mass loss profiles of solid and porous CaP glass microspheres during degradation in ultra purified over 28 days at $37^{\circ} \mathrm{C}$, and $\boldsymbol{c - e}$ ) Cumulative ion release profile $\left(\mathrm{Ca}^{2+}, \mathrm{PO}_{4}^{3-}\right.$, and $\left.\mathrm{Mg}^{2+}\right)$ of solid and porous CaP microspheres in DMEM cell culture medium over 14 days at $37^{\circ} \mathrm{C}$, and $\mathrm{f}$ ) ion release rates (ppm per day) of solid and porous CaP microspheres calculated from the linear cumulative ion release profiles (observed in c-e). 
The biocompatibility evaluation of the porous $\mathrm{CaP}$ glass microspheres was performed through MTT test according to the ISO standard 10993-5. Results revealed that the proliferation rate of hMSCs cultured with the material ion extracts or with 1:10 serial dilutions was higher than 70 $\%$ of the SC medium control $(1 \mathrm{x}: 81 \% \pm 21 ; 1 / 10 \mathrm{x}: 72 \% \pm 16 ; 1 / 100 \mathrm{x}: 103 \% \pm 19 ; 1 / 1000 \mathrm{x}$ : $87 \% \pm 26$ ), as showed in Figure 4a. The suitability of the microspheres to support cell adhesion and growth was also evaluated by culturing cells directly onto the $\mathrm{CaP}$ glass microspheres for up to 14 days in SC medium. Their metabolic activity was analysed at $12 \mathrm{~h}$, day 5 and day 12 which showed a progressive increase of the signal over time, suggesting an increase in cell numbers (see Figure 4b).

a

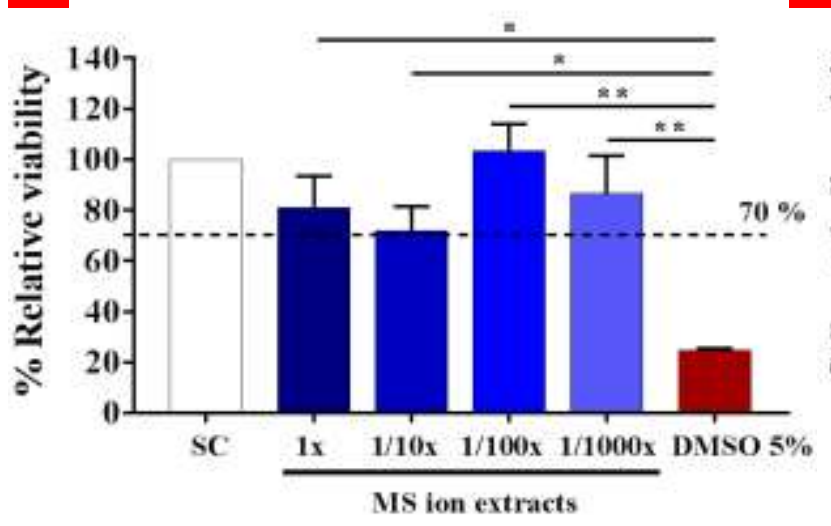

Culture condition

\section{b}

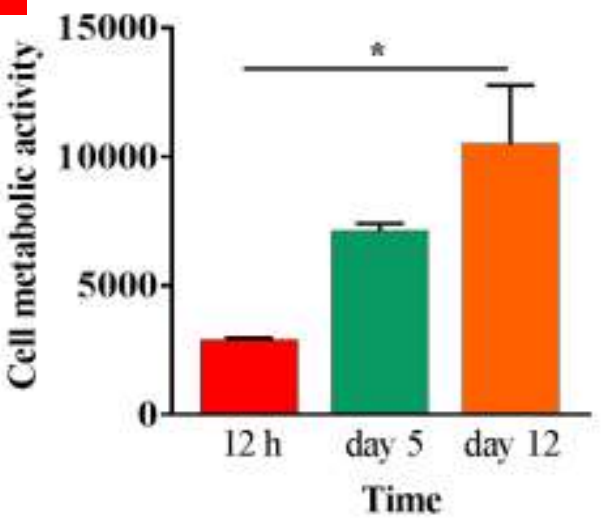

Figure 4: Biocompatibility evaluation of porous CaP glass microspheres according to ISO 10993. a) Results of MTT test showing the relative viability of cells cultured with ion extract conditioned medium (1x) and for the following three serial dilutions (1/10x, 1/100x, 1/1000x). The SC medium only and SC supplemented with $5 \%$ DMSO were included as control. b) Shows the results of cell metabolic activity for porous $\mathrm{CaP}$ microspheres cultured at $12 \mathrm{~h}$, day 5 and day 12 after seeding $(* p<0.05, * * p<0.01)$.

Initial observations confirmed through ESEM imaging showed that the cells were indeed attached and had spread on the microspheres and could also be observed in their inner pore structures (indicated by yellow arrows) where they acquired a 3D conformation (Figure 5a, b).

Further detailed analysis using scanning laser confocal microscopy was performed at day 14, where it was clearly observed that cells were attached to the microspheres and grew on and around them forming macro-aggregates (Figure 5c, d). Moreover, to ascertain how far the stem cells had migrated inside the porous microspheres, z-stack images were taken which confirmed that the cells were present up to half-way inside the porous microspheres (see Figure 5e). 

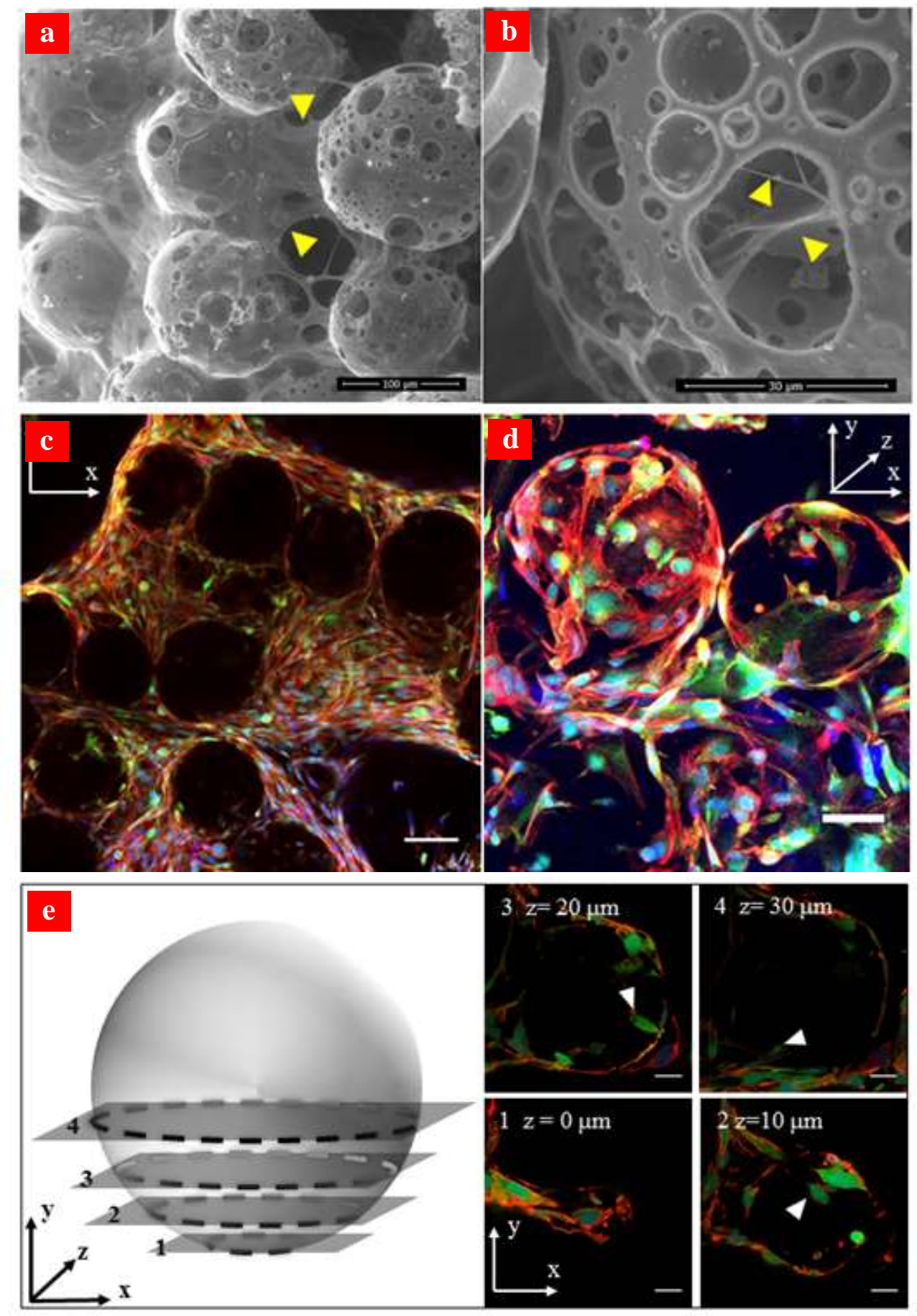

Figure 5: a) ESEM image of stem cell-microsphere aggregates; the yellow arrows indicate hMSCs attached and bridging across several microspheres, b) ESEM image shows colonisation of hMSCs within the pores of the microspheres (yellow arrows), $\boldsymbol{c}, \boldsymbol{d}$ ) Representative confocal images (in single plane and z-stacked mode) of GFP-labelled hMSCs seeded on porous CaP microspheres at day 14, and e) cross sectioned representation of a microsphere and its respective single plane sectioned images; white arrows indicate cells 
which have migrated internally within the microsphere. hMSCs are seen in green (labelled using GFP), the nuclei are stained in blue (Hoechst 33259) and the cytoskeleton is seen in red (Phalloidin), respectively. Scale bars: $100 \mu \mathrm{m}$ (c), $50 \mu \mathrm{m}$ (d) and $25 \mu \mathrm{m}$ (e).

\section{Discussion}

Orthobiologic therapies are being developed to help improve the long-term health of patients suffering from disabling musculoskeletal disorders and is a rapidly advancing field which utilises cell-based therapies and biomaterials to promote healing and offers exciting alternatives to traditional orthopaedic options.

The results above demonstrate (for the first time) a highly successful production process to manufacture highly porous $\mathrm{CaP}$ glass microspheres. The manufacturing route developed relies on a number of key parameters including the chemical and physical interaction of the porogen with the glass the glass viscosity, along with both being delivered within a suitable thermal processing window.

Figure 6 highlights a schematic representation of the morphological changes that occur and the proposed underlying mechanisms to produce the porous glass microspheres. The manufacturing process involved selection of a glass formulation that will not only melt but then allows it to reach a temperature where the viscosity was sufficiently low such that entrapment of the porogen gas could occur within the molten particle, which was itself driven to produce a spherical morphology due to surface tension from being ejected from the flame. The kinetics of decomposition must ideally be sufficiently slow for gas entrapment and adequately fast for pore formation to occur. 


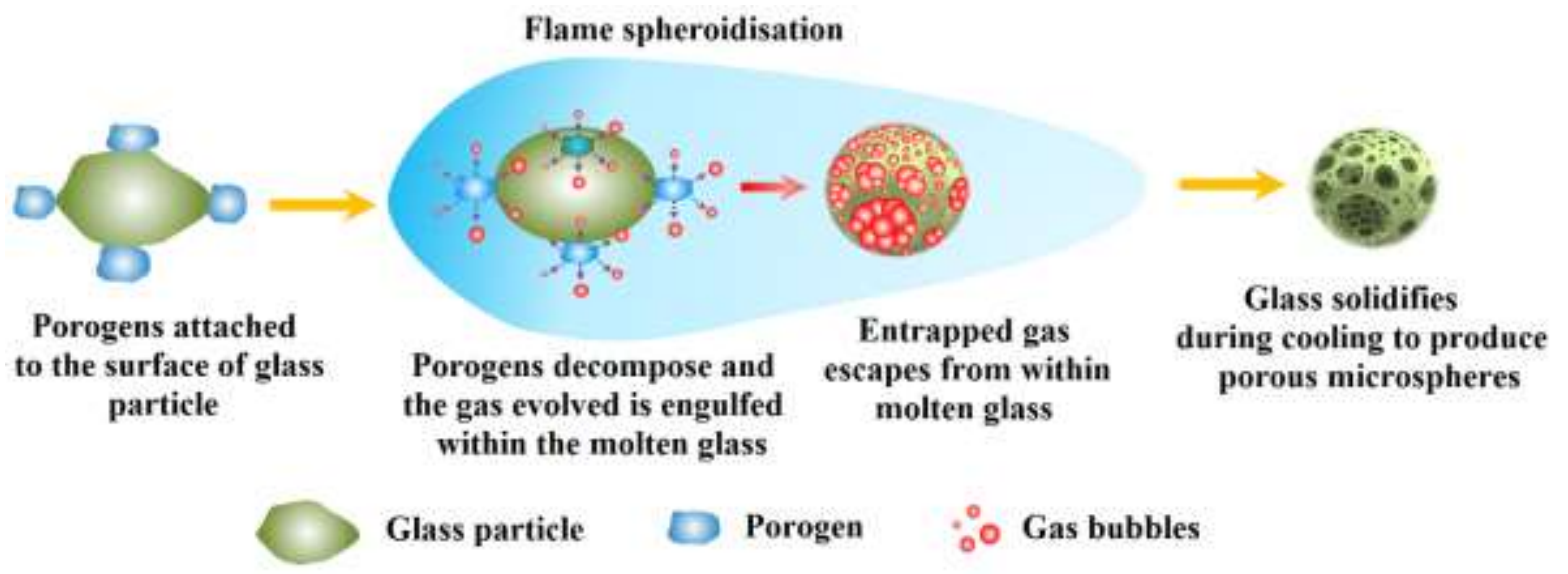

Figure 6: Schematic representation of the proposed mechanism for manufacturing porous glass microspheres via the flame spheroidisation process.

Critical to this process was not only the choice of pore forming agent but also the size of the porogens and in some cases how they were applied to affect the pore sizes and levels produced. As shown in Figure 1, a range of pores, pore sizes and quantities are achievable from this process. Furthermore, it was also found that varying the initial particle size and porogen quantities, enabled control over the size of the surface and interconnected pores.

Examining the $\mathrm{CaP}$ porous glass microspheres reported here the porosity (as measured via mercury intrusion) showed an increase from approximately $37 \%$ for loosely packed non-porous microspheres, which is typical for packed spheres, to over $76 \%$ for the porous microspheres (see Figures $2 \mathrm{a}-\mathrm{c}$ ). These results revealed that the inter-particulate gaps also contributed to the overall porosity levels measured for both the solid (non-porous) and the porous microspheres.

This study also showed that both porogen quantity and microparticles size also effected pore size and surface area, as higher quantities of porogen resulted in increased porosity (see Figures 2c) and smaller (63 $-125 \mu \mathrm{m})$ particle sizes resulted in larger pore sizes. BET analysis confirmed that surface area could also be controlled up to approximately $0.9 \mathrm{~m}^{2} \mathrm{~g}^{-1}$ (see Figures $2 \mathrm{~d}$ ). These values appeared to be low, however the resulting porous microspheres resemble a shell-like structure with fully interconnected pores, which was reflected in the values obtained.

Addition of porogen during the spheroidisation process also resulted in slightly localised increase in Ca content which were mainly observed around the pores (see Figures 3a) and were attributed to the use of Ca-based porogens utilised. The introduction of porosity also resulted in an increase in mass loss during degradation (see Fig 3b) and increased the release of ions as expected due to the increased surface area achieved. As such, by simple selection of glass 
formulations and particle size, the microsphere porosity levels (which in turn relates to their surface area) could easily be manipulated to control ion release rates (as seen in Figures 3c-e) of potentially biotherapeutic ions for specific end applications.

The advantages of larger surface pores are that they could be exploited for applications which require faster ingress of fluids or media through the internal microsphere structure and smaller pores within could be utilised for the entrapment of particulate materials, for example in separation sciences. In particular for biomedical applications, larger pores would be beneficial to accommodate particular cell types, in order to promote tissue engineering or regeneration activities, whereas smaller surface pores could be beneficial for controlling release of drugs and/or other small biological components or biomolecules, entrapped within. Furthermore, where biological cell and material interactions are concerned, the inter-particulate gaps between the microspheres along with the enhanced levels of interconnected porosity created using this simple, single stage manufacturing process, could enable permeation of nutrients and waste products throughout the entire cell/material constructs [42-44].

Recently it has been shown that cell fate, in particular stem cell phenotype, could be influenced by controlled release of specific ions from inorganic materials [45]. Through simple compositional tailoring, the formulation of $\mathrm{CaP}$ glasses can easily be modified to release ions of specific interest. The solid and porous microspheres manufactured in this study revealed release of $\mathrm{Ca}^{2+}, \mathrm{PO}_{4}{ }^{3-}$ and $\mathrm{Mg}^{2+}$ ions ranging from 45-47, 34-49 and 13-20 ppm per day (see Figure 3f). Furthermore, studies by Ahmed et al [46], showed that phosphate anionic species (ranging from ortho, pyro, meta and polyphosphates) could also be controlled, by simple tailoring of the starting glass formulations.

Figure 7 highlights some of the ions which could be released from $\mathrm{CaP}$ glasses and their potential biotherapeutic roles in bone repair and regeneration. $\mathrm{Ca}^{2+}$ ions are known to stimulate proliferation and differentiation of osteoblasts as well as extracellular matrix mineralisation [47] whilst $\mathrm{Mg}^{2+}$ ions can promote new bone formation [48]. Moreover, $\mathrm{PO}_{4}{ }^{3-}$ ions are required for calcium phosphate crystal deposition [49] and extracellular matrix mineralisation while $\mathrm{Na}^{+}$ ions are mainly found in extracellular fluid [50]. 

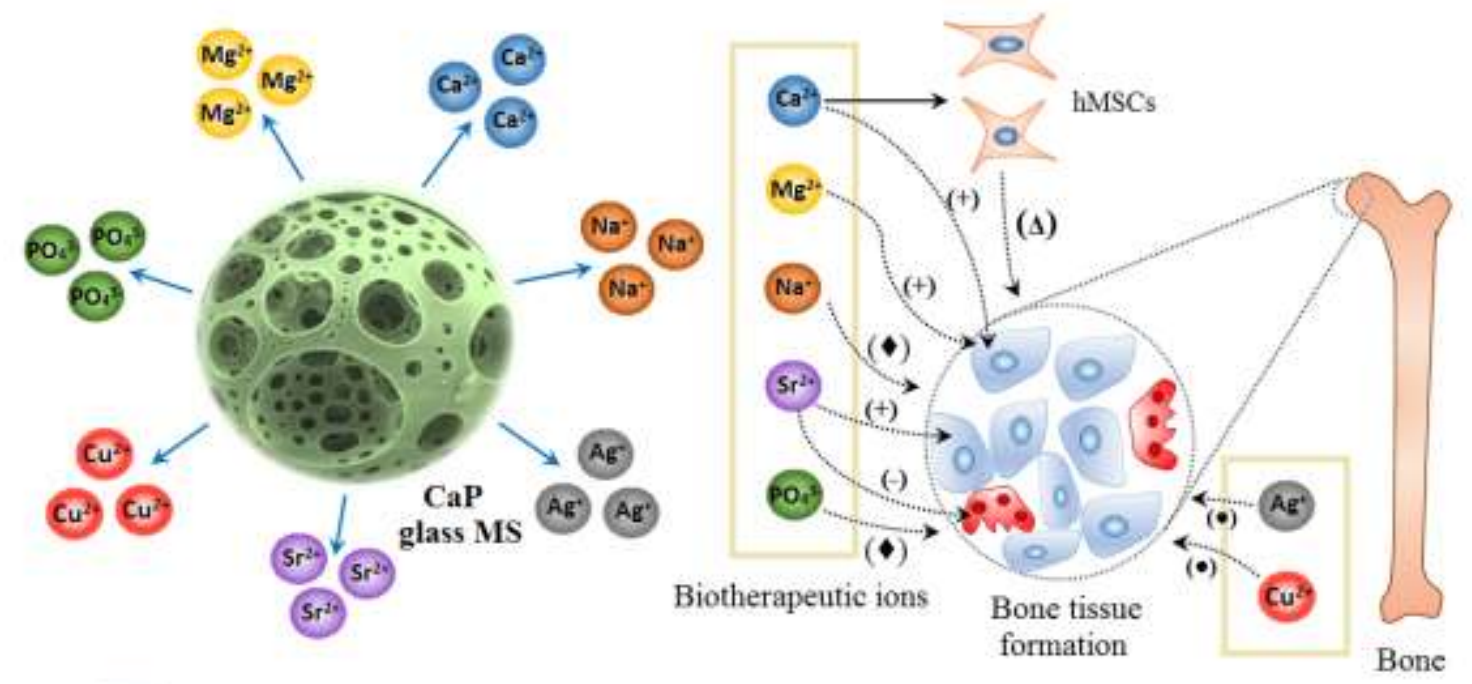
Osteoblasts
(+) Stimulate bone formation
(४) Promote bone mineralisation
(-) Inhibit bone resorption
(A) Stimulate osteogenesis
Osteoclasts
(•) Combat antibacterial infection during bone regeneration

Figure 7: Biotherapeutic ions which can be released from CaP glass and their role in bone tissue formation.

Other biotherapeutic ions can also be released from the CaP microspheres such as $\mathrm{Sr}^{2+}$ [12], $\mathrm{Cu}^{2+}$ [9] and $\mathrm{Ag}^{+[10]}$ by simply doping the glass composition with the desired metal oxide. For instance, $\mathrm{Sr}^{2+}$ ions are known to inhibit osteoclast activity whilst promoting osteogenesis of mesenchymal stem cells in vitro and in vivo [51], $\mathrm{Ag}^{2+}$ and $\mathrm{Cu}^{2+}$ ions have both demonstrated antimicrobial properties while $\mathrm{Cu}^{2+}$ ions have been also shown to promote the proliferation of endothelial growth factors and fibroblast growth factor 2 (FGF-2), which are associated with the angiogenesis process [9].

This study also demonstrated that the porous microspheres produced were biocompatible (see Figure 4a) according to the standard ISO 10993-5 (i.e. used for the biological evaluation of biomedical devices). Moreover, when the cells were seeded and cultured directly onto the microspheres, they were not only able to attach but had also migrated to within the centre of the microspheres, revealing colonisation of the inner pore structures (see Figure 5e). The pores formed presented the cells with a high surface area and a niche environment for the cells to grow and proliferate, acting as sites for cell culture [52]. This niche pore environment could also reduce any potential shear stresses experienced by the cells and provide protection for cells from getting damaged via inter-particle abrasion. Furthermore, the pores also closely resemble the native $3 \mathrm{D}$ conformation of bone tissue [53]. It has also been proposed that a porous 
environment would favour the capture and utilisation of cell secreted factors close to where the cells reside in order to further influence cell phenotype [45].

The porous microspheres developed in this study constitute a versatile and highly promising technology for wider applications in tissue engineering and regenerative medicine (with a particular focus in bone regeneration) as initial examples. Indeed, doping the starting glass formulations with specific biotherapeutic ions could also address other key aspects of bone regeneration to include angiogenesis, osteogenesis and control of osteoclast activity, all potentially also coupled with antimicrobial properties. Further studies are on-going aimed at investigating more in-depth the bone tissue regenerative potential of these microspheres as well as exploring their potential use in alternate biomedical applications.

The porous glass microsphere production process developed herein offers a new cost-effective single stage manufacturing process, with demonstrated scale-up potential and with production levels in the range of kg's per hour at lab scale, which could easily be further scaled-out to commercial scales. Follow-on investigations are on-going to explore utility of this manufacturing process for other glass types (i.e. borates and silicates including glass-ceramics).

\section{Conclusions}

This study highlights a novel flame spheroidisation process to manufacture highly porous $\mathrm{CaP}$ glass microspheres. Microspheres with a range of surface and fully interconnected porosity from $52( \pm 5)$ to $76( \pm 5) \%$ were manufactured by simply manipulating process parameters such as the ratios of glass particles to porogen used (from 1:1 to 1:3) including the oxy-acetylene gas ratios.

The increase in porosity levels of the microspheres was shown to accelerate degradation over a 28 day degradation period (i.e. a 30\% mass loss was observed for porous microspheres, compared to a $9 \%$ mass loss for the solid non-porous microspheres). The ion release profiles of the porous microspheres also revealed increasing rates as expected, compared to the nonporous microspheres, which was attributed to their increased surface area profiles.

The cell culture study revealed that the ion extracts from the $\mathrm{CaP}$ microspheres (utilising indirect cell culture method according to ISO 10993) did not exert any negative effect on the stem cells (hBM-MSCs). In addition, the direct cell culture study revealed that the porous 
microspheres supported cell attachment as well as proliferation and showed that the hBMMSCs had migrated into and towards the centre of the microspheres. The pore sizes and porosity levels achieved opens up the potential for use of these porous $\mathrm{CaP}$ microspheres for other biomedical applications in addition to the proposed orthobiologic applications.

\section{Acknowledgements:}

This work has been funded through the RDF-PP-0303 (University of Nottingham), IKC University of Leeds (EP/G032483/1), EPSRC IAA (University of Nottingham, ref. no. EP/K503800/1), Science and Technology Facilities Council (ST/L502583/1) and the National Institute for Health Research (NIHR) Invention for Innovation (i4i) Challenge Award Programme (II-C3-0714-20001). The views expressed above are those of the authors and not necessarily those of the NHS, the NIHR or the Department of Health.

The authors would also like to acknowledge Nicola Weston at the Nanoscale and Microscale Research Centre (NMRC) at the University of Nottingham for use of the electron microscope facilities and Dr Christhopher Gell of the University of Nottingham's School of Life Sciences Imaging (SLIM) for the use of confocal microscopy and help with image analysis. 


\section{References:}

[1] A. Weglein, S. Sampson, D. Aufiero, Platelet Rich Plasma Practical Use in Non-Surgical Musculoskeletal Pathology, in: J.F.S.D. Lana, M.H. Andrade Santana, W. Dias Belangero, A.C. Malheiros Luzo (Eds.), Platelet-Rich Plasma: Regenerative Medicine: Sports Medicine, Orthopedic, and Recovery of Musculoskeletal Injuries, Springer Berlin Heidelberg, Berlin, Heidelberg, 2014, pp. 187-201.

[2] S. Sampson, A.B.-v. Bemden, D. Aufiero, Autologous Bone Marrow Concentrate: Review and Application of a Novel Intra-Articular Orthobiologic for Cartilage Disease, The Physician and Sportsmedicine 41(3) (2013) 7-18.

[3] B.C. Toolan, Current Concepts Review: Orthobiologics, Foot \& Ankle International 27(7) (2006) 561-566.

[4] L.L. Hench, J.R. Jones, Bioactive Glasses: Frontiers and Challenges, Frontiers in Bioengineering and Biotechnology 3(194) (2015).

[5] L.L. Hench, The story of Bioglass ${ }^{\circledR}$, Journal of Materials Science: Materials in Medicine 17(11) (2006) 967-978.

[6] X. Zhang, D. Zeng, N. Li, J. Wen, X. Jiang, C. Liu, Y. Li, Functionalized mesoporous bioactive glass scaffolds for enhanced bone tissue regeneration, Scientific Reports 6 (2016) 19361.

[7] J.R. Jones, L.M. Ehrenfried, L.L. Hench, Optimising bioactive glass scaffolds for bone tissue engineering, Biomaterials 27(7) (2006) 964-973.

[8] W. Huang, D.E. Day, K. Kittiratanapiboon, M.N. Rahaman, Kinetics and mechanisms of the conversion of silicate (45S5), borate, and borosilicate glasses to hydroxyapatite in dilute phosphate solutions, Journal of Materials Science: Materials in Medicine 17(7) (2006) 583-596.

[9] E.A. Abou Neel, I. Ahmed, J. Pratten, S.N. Nazhat, J.C. Knowles, Characterisation of antibacterial copper releasing degradable phosphate glass fibres, Biomaterials 26(15) (2005) 2247-2254.

[10] I. Ahmed, E.A. Abou Neel, S.P. Valappil, S.N. Nazhat, D.M. Pickup, D. Carta, D.L. Carroll, R.J. Newport, M.E. Smith, J.C. Knowles, The structure and properties of silver-doped phosphate-based glasses, Journal of Materials Science 42(23) (2007) 9827-9835.

[11] E.A. Abou Neel, W. Chrzanowski, J.C. Knowles, Effect of increasing titanium dioxide content on bulk and surface properties of phosphate-based glasses, Acta Biomaterialia 4(3) (2008) 523-534.

[12] U. Patel, R.M. Moss, K.M.Z. Hossain, A.R. Kennedy, E.R. Barney, I. Ahmed, A.C. Hannon, Structural and physico-chemical analysis of calcium/strontium substituted, near-invert phosphate based glasses for biomedical applications, Acta Biomaterialia 60(Supplement C) (2017) 109-127. [13] I. Ahmed, A. Parsons, A. Jones, G. Walker, C. Scotchford, C. Rudd, Cytocompatibility and Effect of Increasing MgO Content in a Range of Quaternary Invert Phosphate-based Glasses, Journal of Biomaterials Applications 24(6) (2010) 555-575.

[14] E.A. Abou Neel, L.A. O'Dell, W. Chrzanowski, M.E. Smith, J.C. Knowles, Control of surface free energy in titanium doped phosphate based glasses by co-doping with zinc, J Biomed Mater Res B Appl Biomater. 2009 May;89(2):392-407.

[15] M. Bitar, V. Salih, J.C. Knowles, M.P. Lewis, Iron-phosphate glass fiber scaffolds for the hard-soft interface regeneration: the effect of fiber diameter and flow culture condition on cell survival and differentiation, J Biomed Mater Res A. 2008 Dec 15;87(4):1017-26.

[16] N.J. Lakhkar, E.A. Abou Neel, V. Salih, J.C. Knowles, Strontium oxide doped quaternary glasses: effect on structure, degradation and cytocompatibility, J Mater Sci Mater Med. 2009 Jun;20(6):133946. Epub 2009 Jan 10.

[17] S.P. Valappil, D.M. Pickup, D.L. Carroll, C.K. Hope, J. Pratten, R.J. Newport, M.E. Smith, M. Wilson, J.C. Knowles, Effect of silver content on the structure and antibacterial activity of silverdoped phosphate-based glasses, Antimicrob Agents Chemother. 2007 Dec;51(12):4453-61. Epub 2007 Oct 1. 
[18] S.P. Valappil, D. Ready, E.A. Abou Neel, D.M. Pickup, L.A. O'Dell, W. Chrzanowski, J. Pratten, R.J. Newport, M.E. Smith, M. Wilson, J.C. Knowles, Controlled delivery of antimicrobial gallium ions from phosphate-based glasses, Acta Biomater. 2009 May;5(4):1198-210. Epub 2008 Oct 10.

[19] I. Ahmed, M. Lewis, I. Olsen, J.C. Knowles, Phosphate glasses for tissue engineering: Part 2.

Processing and characterisation of a ternary-based P2O5-CaO-Na2O glass fibre system, Biomaterials 25(3) (2004) 501-7.

[20] I. Ahmed, M. Lewis, I. Olsen, J.C. Knowles, Phosphate glasses for tissue engineering: Part 1. Processing and characterisation of a ternary-based P2O5-CaO-Na2O glass system, Biomaterials 25(3) (2004) 491-499.

[21] S. Mitragotri, P.A. Burke, R. Langer, Overcoming the challenges in administering biopharmaceuticals: formulation and delivery strategies, Nat Rev Drug Discov 13(9) (2014) 655-672. [22] V.V. Budov, Hollow glass microspheres. use, properties, and technology (Review), Glass and Ceramics 51(7-8) (1994) 230-235.

[23] S. Labbaf, O. Tsigkou, K.H. Müller, M.M. Stevens, A.E. Porter, J.R. Jones, Spherical bioactive glass particles and their interaction with human mesenchymal stem cells in vitro, Biomaterials 32(4) (2011) 1010-1018.

[24] X. Zhao, S. Liu, L. Yildirimer, H. Zhao, R. Ding, H. Wang, W. Cui, D. Weitz, Injectable Stem CellLaden Photocrosslinkable Microspheres Fabricated Using Microfluidics for Rapid Generation of Osteogenic Tissue Constructs, Advanced Functional Materials 26(17) (2016) 2809-2819.

[25] K.J. Pekarek, J.S. Jacob, E. Mathiowitz, Double-walled polymer microspheres for controlled drug release, Nature 367(6460) (1994) 258-260.

[26] X. Liu, X. Jin, P.X. Ma, Nanofibrous hollow microspheres self-assembled from star-shaped polymers as injectable cell carriers for knee repair, Nat Mater 10(5) (2011) 398-406.

[27] W. Li, J.Y. Walz, Porous Nanocomposites with Integrated Internal Domains: Application to Separation Membranes, Sci. Rep. 4 (2014).

[28] Z.-C. Yang, C.-H. Tang, Y. Zhang, H. Gong, X. Li, J. Wang, Cobalt monoxide-doped porous graphitic carbon microspheres for supercapacitor application, Sci. Rep. 3 (2013).

[29] Y.N. Ko, S.B. Park, S.H. Choi, Y.C. Kang, One-pot synthesis of manganese oxide-carbon composite microspheres with three dimensional channels for Li-ion batteries, Sci. Rep. 4 (2014).

[30] S. Li, L. Nguyen, H. Xiong, M. Wang, T.C.C. Hu, J.-X. She, S.M. Serkiz, G.G. Wicks, W.S. Dynan, Porous-wall hollow glass microspheres as novel potential nanocarriers for biomedical applications, Nanomedicine: Nanotechnology, Biology and Medicine 6(1) (2010) 127-136.

[31] H.X. Peng, Z. Fan, J.R.G. Evans, J.J.C. Busfield, Microstructure of ceramic foams, Journal of the European Ceramic Society 20(7) (2000) 807-813.

[32] L. Montanaro, Y. Jorand, G. Fantozzi, A. Negro, Ceramic foams by powder processing, Journal of the European Ceramic Society 18(9) (1998) 1339-1350.

[33] D. Enke, R. Gläser, U. Tallarek, Sol-Gel and Porous Glass-Based Silica Monoliths with Hierarchical Pore Structure for Solid-Liquid Catalysis, Chemie Ingenieur Technik 88(11) (2016) 1561-1585.

[34] Z.Y. Wu, R.G. Hill, S. Yue, D. Nightingale, P.D. Lee, J.R. Jones, Melt-derived bioactive glass scaffolds produced by a gel-cast foaming technique, Acta Biomaterialia 7(4) (2011) 1807-1816.

[35] Q. Fu, E. Saiz, A.P. Tomsia, Bioinspired Strong and Highly Porous Glass Scaffolds, Adv Funct Mater 21(6) (2011) 1058-1063.

[36] S. Hyuk Im, U. Jeong, Y. Xia, Polymer hollow particles with controllable holes in their surfaces, Nat Mater 4(9) (2005) 671-675.

[37] S.-W. Choi, Y. Zhang, Y.-C. Yeh, A. Lake Wooten, Y. Xia, Biodegradable porous beads and their potential applications in regenerative medicine, Journal of Materials Chemistry 22(23) (2012) 1144211451.

[38] M. Bohner, S. Tadier, N. van Garderen, A. de Gasparo, N. Döbelin, G. Baroud, Synthesis of spherical calcium phosphate particles for dental and orthopedic applications, Biomatter 3(2) (2013) e25103. 
[39] H.-H. Lee, S.-J. Hong, C.-H. Kim, E.-C. Kim, J.-H. Jang, H.-I. Shin, H.-W. Kim, Preparation of hydroxyapatite spheres with an internal cavity as a scaffold for hard tissue regeneration, Journal of Materials Science: Materials in Medicine 19(9) (2008) 3029-3034.

[40] M. Kawashita, S. Toda, H.-M. Kim, T. Kokubo, N. Masuda, Preparation of antibacterial silverdoped silica glass microspheres, Journal of Biomedical Materials Research Part A 66A(2) (2003) 266274.

[41] N.J. Lakhkar, J.-H. Park, N.J. Mordan, V. Salih, I.B. Wall, H.-W. Kim, S.P. King, J.V. Hanna, R.A. Martin, O. Addison, J.F.W. Mosselmans, J.C. Knowles, Titanium phosphate glass microspheres for bone tissue engineering, Acta Biomaterialia 8(11) (2012) 4181-4190.

[42] Y. Senuma, S. Franceschin, J.G. Hilborn, P. Tissiéres, P. Frey, Bioresorbable Microspheres as Injectable Cell Carrier: FRom Preparation to in Vitro Evaluation, MRS Proceedings 550 (1998). [43] O. Qutachi, J.R. Vetsch, D. Gill, H. Cox, D.J. Scurr, S. Hofmann, R. Müller, R.A. Quirk, K.M. Shakesheff, C.V. Rahman, Injectable and porous PLGA microspheres that form highly porous scaffolds at body temperature, Acta Biomaterialia 10(12) (2014) 5090-5098.

[44] Y. Cai, Y. Chen, X. Hong, Z. Liu, W. Yuan, Porous microsphere and its applications, International Journal of Nanomedicine 8 (2013) 1111-1120.

[45] W.L. Murphy, T.C. McDevitt, A.J. Engler, Materials as stem cell regulators, Nat Mater 13(6) (2014) 547-557.

[46] I. Ahmed, M.P. Lewis, S.N. Nazhat, J.C. Knowles, Quantification of Anion and Cation Release from a Range of Ternary Phosphate-based Glasses with Fixed 45 mol\% P2O5, Journal of Biomaterials Applications 20(1) (2005) 65-80.

[47] S. Maeno, Y. Niki, H. Matsumoto, H. Morioka, T. Yatabe, A. Funayama, Y. Toyama, T. Taguchi, J. Tanaka, The effect of calcium ion concentration on osteoblast viability, proliferation and differentiation in monolayer and 3D culture, Biomaterials 26(23) (2005) 4847-55.

[48] S. Yoshizawa, A. Brown, A. Barchowsky, C. Sfeir, Role of magnesium ions on osteogenic response in bone marrow stromal cells, Connect Tissue Res 55 (2014) 155-159.

[49] M. Julien, S. Khoshniat, A. Lacreusette, M. Gatius, A. Bozec, E.F. Wagner, Y. Wittrant, M. Masson, P. Weiss, L. Beck, D. Magne, J. Guicheux, Phosphate-Dependent Regulation of MGP in Osteoblasts: Role of ERK1/2 and Fra-1, Journal of Bone and Mineral Research 24(11) (2009) 18561868.

[50] D.I. Hamasaki, The effect of sodium ion concentration on the electroretinogram of the isolated retina of the frog, The Journal of Physiology 167(1) (1963) 156-168.

[51] V. Mouriño, J.P. Cattalini, A.R. Boccaccini, Metallic ions as therapeutic agents in tissue engineering scaffolds: an overview of their biological applications and strategies for new developments, Journal of The Royal Society Interface 9(68) (2012) 401-419.

[52] D. Looby, B. Griffiths, Immobilization of animal cells in porous carrier culture, Trends in Biotechnology 8 204-209.

[53] L. Andrea Di, L. Alessia, C. Giuseppe, M. Carlos, B. Clemens van, M. Lorenzo, Toward mimicking the bone structure: design of novel hierarchical scaffolds with a tailored radial porosity gradient, Biofabrication 8(4) (2016) 045007. 


\section{Graphical abstract}

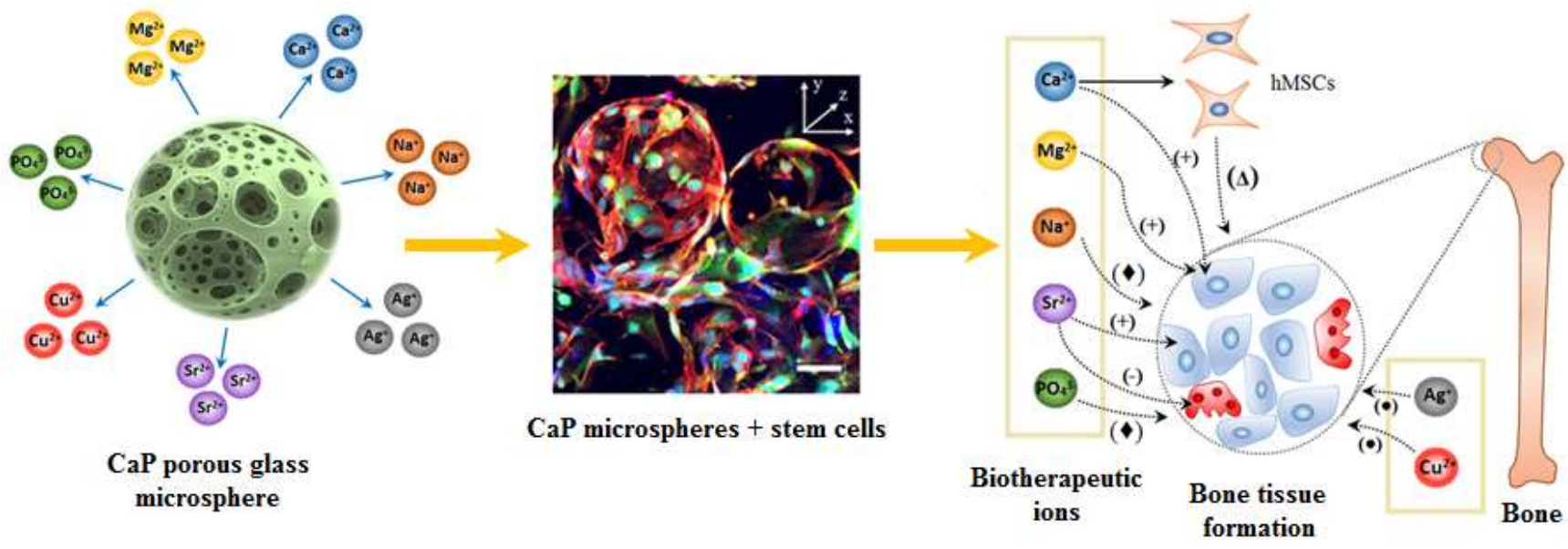

